\title{
Faecal Transplantation and Clostridioides difficile Infection
}

\author{
Darija A Knežević, ${ }^{1}$ Miroslav Petković
}

\begin{abstract}
Faecal microbiota transplantation (FMT), known equally well as faecal transplantation or faecal bacteriotherapy, is the process of implanting the faecal suspension containing balanced microbiota from a healthy donor to the colon of a recipient patient. Excessive growth of Clostridioides difficile ( $C$ difficile) in the intestinal microbiota resulting from antibiotic consumption is currently a rising threat to public health. FMT is one of the most important, newer approaches to treating $C$ difficile infections. Since $C$ difficile is regarded as an opportunistic bacterium triggering disease in conditions of disturbed homeostasis of the intestinal microbiota, restoration of healthy intestinal microflora facilitates suppression of toxic strain of $C$ difficile by anaerobic bacteria of normal intestinal microflora with concomitant cure. Nurses have important role in caring for patients after faecal transplantation.
\end{abstract}

Key words: Faecal microbiota transplantation; Clostridioides difficile infection; Microbiota.
(1) Department of Nursing, Faculty of Medicine, University of Banja Luka, Banja Luka, the Republic of Srpska, Bosnia and Herzegovina.

(2) Department of Microbiology and Immunology, Faculty of Medicine, University of Banja Luka, Banja Luka, the Republic of Srpska, Bosnia and Herzegovina.

Correspondence:

DARIJA A KNEŽEVIĆ

T: +38751923633

E: darija.a.knezevic@med.unibl.org

ARTICLE INFO

Received: 15 June 2021 Revision received: 22 August 2021 Accepted: 24 August 2021

\section{Introduction}

Faecal microbiota transplantation (FMT), also called faecal transplantation or faecal bacteriotherapy, is the transfer of a faecal suspension from a healthy donor into the colon of a recipient patient. The goal of FMT is to achieve the restoration of the intestinal flora of an infected person by transmitting the intestinal flora bacteria of a healthy donor. FMT is one of the more important, newer approaches for the treatment of Clostridioides difficile ( $C$ difficile) infections (CDI) $\cdot^{1-3}$ As a method of treatment, FMT was used long before modern medicine. Ancient civilisations did not know the concept of microbiota but talked about the healing effect of faeces. The first recorded case of ingestion of faecal material for medical reasons dates from the traditional Chinese medicine that in the $4^{\text {th }}$ century used this procedure for the treatment of severe diarrhoea, food poisoning and malaria (recorded in the book Prescription Collection of Emergency). This treatment is also mentioned by Li Shizhen in the $16^{\text {th }}$ century in his famous book
Ben Cao Gang Mu (Compendium of Materia Medica) for the treatment of gastrointestinal diseases as a "yellow soup"., 5 In modern medicine, the first published study in English literature of the use of FMT dated from 1958 and talked about the use of faecal enema, as an additional procedure for pseudomembranous colitis caused by antibiotics. It is rarely mentioned later, until in 2010 when FMT has been increasingly recognised as a successful therapeutic procedure for recurrent cases of CDI, but also the treatment of ulcerative colitis, Crohn's disease, irritable bowel syndrome, metabolic syndrome, and even chronic fatigue syndrome. ${ }^{6-9}$

\section{Microbiota and microbiome}

More than 100 billion symbiotic microorganisms are found in and on humans, which is on average 
ten times more than the estimated number of cells in the human body. Most of these microorganisms inhabit the gastrointestinal tract (GT), which contains about 150 times more genes (microbiome) than are identified in the human genome, so this complex community of GT is called the intestinal microbiota. More than 1,000 separate species of bacteria have been discovered in the human microbiota using molecular diagnostic techniques, many of which cannot be cultured. ${ }^{10-13}$ Humans live with their microbiota forming a complex symbiotic system in which balance (eubiosis) and microbiota disorder (dysbiosis) favour the development of some chronic diseases (eg, allergic diseases, obesity, diabetes mellitus, metabolic syndrome, atherosclerosis, malignant diseases, inflammatory bowel disease). ${ }^{14,15}$

The physiological microbiota of an individual is rapidly formed during the first months of life. By the end of the second year, it achieves a composition that remains stable throughout life. Interaction with the intestinal microbiota in infancy plays an essential role in health and disease in later life, creating immune and metabolic pathways. During birth and after birth, the newborn's body is being quickly inhabited by microorganisms with which it comes into contact. ${ }^{16-18}$ Data from epidemiological studies have shown that caesarean section, preterm birth, early exposure to antibiotics, and supplementation with adapted milk harm the development of the intestinal microbiota and contribute to some diseases later in life, for example obesity, asthma and inflammatory bowel disease. ${ }^{19}$

Although no two humans with the same microbiota exist, there are more remarkable similarities in their composition among members of the same race, ethnic groups and related individuals. ${ }^{20,21}$ The type and function of the human microbiota differ depending on the anatomical localisation, age, sex, race and diet of the host. Bacteria are primarily present in a healthy human microbiota. However, in addition to them, some viruses (primarily bacteriophages), fungi, archaea (mainly of the genus Methanobrevibacter) and some eukaryotes are also present. The most significant number of microorganisms of the human microbiota is found in the intestines, greatly influencing the formation of the metabolic phenotype, the epithelium's development, and innate immunity. ${ }^{22-24}$ Bacteria that predominate in the colon are Bacteroidetes (including the genus Bacteroides) and Firmicutes (including the genera Clostridioides and
Eubacterium). Actinobacteria, Verrucomicrobia, Proteobacteria and Fusobacteria appear as well in smaller numbers. ${ }^{25},{ }^{26}$ Intestinal commensals prevent colonisation by pathogenic microorganisms by occupying living space and utilising food and in several indirect ways, such as stimulating a local immune response or producing various compounds with an antimicrobial role. Thus, for example, lactic acid produced by the species of the genus Lactobacillus participates in the degradation of the bacterial wall, and the constitution of bile acids, which reflects the overall metabolism of the intestinal microbiota, significantly affects the intergrowth of $C$ difficile spores. ${ }^{27}$

Bacteria, human symbiotes, aid in the metabolism of difficult-to-digest compounds by providing the host with essential nutrients, prevent expansion of opportunistic microorganisms and add to the creation of intestinal architectures. Foods that are difficult to digest, such as dietary fibre xyloglucan, usually found in vegetables, can be broken down by bacteria from the genus Bacteroides. Bacteria from the genera Lactobacillus and Bifidobacterium enable the digestion of difficult-to-digest fibres, for instance fructooligosaccharides. The gut flora alters the metabolism of carbohydrates, proteins and lipids. With its enzymes, it affects the host enzymes by fermentation. Around 50-100 mmol/L of the short chain fatty acids (SCFA), notably acetic, propionic and butyric acid, are produced daily by intestinal microbiota, thus providing the source of energy for the intestinal epithelium.

The microbiota carries an indispensable role in the synthesis of folate, vitamin $\mathrm{K}$ and some $\mathrm{B}$ vitamins, such as biotin (B1), riboflavin (B2) and cobalamin (B12). It also participates in the metabolism of bile acids, polyphenols, xenobiotics and drugs. In addition, bacteria that colonise the intestine promote the proper development of the mucosal immune system. ${ }^{28,29}$ Alterations in the intestinal microbiota have been observed in obesity, irritable bowel syndrome, ulcerative colitis, Crohn's disease, inflammatory bowel disease, colorectal cancer, non-alcoholic fatty liver, and some other diseases. ${ }^{30-32}$ Since most primary bacterial pathogens have been successfully brought under control by vaccination or improved living conditions, today, the onset of bacterial disease is more associated with disruption of a complex human ecosystem than with the pathogenic power of a single microorganism. Given the high variability of the human microbiota, it is difficult to deter- 
mine what a normal or healthy microbiota is. The ability to treat or prevent disease by altering an individual's microbiota is a significant challenge in many areas of medicine. ${ }^{33}$

\section{Intestinal microbiota and infec- tious diseases}

The intestinal microbiota is made up of microorganisms, which inhabit various regions of the gastrointestinal tract. The number of bacteria increases towards the end of the digestive tract. The stomach and duodenum are settled by a small number of microorganisms, less than $10^{3}$ colony-forming units (CFU) per gram of intestinal contents. Infectious disease is most often the result of microbiota dysbiosis. Then again, infection and its treatment directly influence the intestinal microbiota, which determines the outcome of infection in the human population. The spread of some opportunistic (Enterococcus faecalis, Enterococcus faecium, and bacteria of the Enterobacteriaceae family) and pathogenic bacteria (Salmonella enterica, $C$ difficile) results in a robust inflammatory response, followed by a change in the intestinal microbiota. ${ }^{34-36}$ Numerous studies have demonstrated an association between infection and microbiota dysbiosis. The intestinal microbiota of patients with CDI was significantly altered. ${ }^{37,38}$ Also, microbiota disorders lead to the advancement of infection with viruses such as hepatitis $B$ virus (HBV) or human immunodeficiency virus (HIV). ${ }^{39-41}$

\section{Clostridioides difficile infection}

Excessive growth of $C$ difficile bacterium in the intestinal microbiota gives rise to diarrhoea. It is one of the most common complications that occur after the administration of antibiotics, currently presenting the rising threat to public health. Antibiotics disrupt the homeostasis of the intestinal mucosa, thus reducing resistance to the effect of $C$ difficile toxin and leading to the progression of CDI. The higher incidence of these infections in recent years has been closely related to the development of highly virulent strains, such as those with ribotype 027 (NAP1 / 027 / BI). ${ }^{42,43}$ C difficile is an anaerobic, sporogenic, rod-shaped, Gram-positive bacterium. It was discovered in 1935 in newborns and was named Bacillus difficilis (lat. Difficilis heavy) due to its difficult cultivation. Later, due to the phenotypic similarity with bacteria from the genus Clostridium, it was named Clostridium difficile. Since 2016, based on the sequence analysis of $16 \mathrm{~S}$ rRNA gene, it has been called Clostridioides difficile. ${ }^{44-46}$ It is omnipresent in nature, inhabiting the intestines of humans and animals as well. The proliferation of toxigenic strains of this bacterium leads to various clinical conditions, from asymptomatic carriers, through diarrhoea and self-limiting colitis to serious situations just as fulminant colitis and toxic megacolon. ${ }^{47}$ CDI transmission is horizontal, faecal-oral. In healthcare facilities, it is usually transmitted by contaminated hands (healthcare workers, patients and their families) and objects from the environment (stethoscopes, thermometers, surfaces and space near the patient)..$^{48}$ Although it can be found in meat and meat products, seafood, and fresh vegetables and fruits, no food-borne epidemic disease is directly related to $C$ difficile. ${ }^{49,50}$ Thanks to the resistance of its spores (they tolerate most antiseptics), $C$ difficile can persist for a long time in the external environment, which allows it to stay in the patient's vicinity for a long time. It can be found on various surfaces in the hospital with which patients come into contact and on the hands, clothes, and shoes of medical staff, which according to research, has one of the essential roles in the spread of infection. ${ }^{51}$ Risk factors for the occurrence of the disease are old age, severe chronic diseases, long-term hospitalisation, as well as staying in institutions for the elderly and the chronically ill. However, the most critical factor of risk contributing to the expansion of infection is the change in the intestinal microbiota due to antibiotic therapy. The highest risk is therapy with clindamycin, cephalosporins, penicillins and fluoroquinolones. Moderate risk involves therapy with macrolides and sulphonamides, while treatment with tetracyclines carries no risk. ${ }^{52}$

$C$ difficile is ubiquitous and is statistically present in $15-70 \%$ of neonates and in 2-5\% of the adult population, who are mostly colonised by its community. For example, in most neonates colonisation with $C$ difficile is temporarily, and colitis does not develop. ${ }^{53}$ The observed transient asymptomatic colonisation might result from $C$ difficile toxin-binding receptors, a disorder of antibody production against $C$ difficile toxins, and the development of protective mechanisms associated with breastfeeding or intestinal bile acid me- 
tabolism. ${ }^{54,55}$ It is recommended not to perform diagnostic procedures, nor therapy treatments in patients who are asymptomatic, as antimicrobial agents may cause unwanted changes in composition of gut microbiota. ${ }^{56,57}$ Recognition of the symptoms of CDI in adult patients is critical for timely initiation of therapy. Wide range of CDI symptoms are documented, from mild diarrhoea as a the most common symptom, to severe form of ulcerative colitis leading to toxic megacolon or to the perforation of the colon. ${ }^{58}$ Usually patients with confirmed CDI do not have diarrhoea with blood (bloody diarrhoea), which can be found in patients with inflammatory bowel disease. ${ }^{59}$ The rate of first recurrent CDI cases is between $10 \%$ and $20 \%$. A recurrent case of CDI is identified as an infection that reoccurs within eight weeks after the end of the last episode of the disease. With each subsequent repetition of CDI, the repetition rate increases significantly. After the first relapse, the cash recurrence rate increases to $40 \%$ and then to more than $60 \%$ for each subsequent period.

Patients with recurrent CDI have a deficiency of bacteria prevalent in the colon, leading to multiple relapses. ${ }^{60,61}$ When repeated CDI is diagnosed, it is recommended to treat the first CDI relapse with the same therapy as the one used for the initial case of infection (metronidazole or vancomycin). Nevertheless, many authors suggest vancomycin for the treatment of the first relapse after initial infection with CDI was treated with metronidazole. If the first case of CDI was treated orally with vancomycin, fidaxomicin is the next choice of therapy for the first relapse, although it is often budget limited. Since there are concerns about the occurrence of neurotoxic effects with long-term use, metronidazole is not recommended as a therapy for the second relapse. Moreover, the effect of metronidazole is reduced after repeated infections. Thus, for any subsequent relapse, the therapy with antibiotics for a more extended period, even longer than four weeks, often with reduced or increased doses of vancomycin, is considered..$^{62}$ Due to the limited possibilities of medical treatment of recurrent cases of CDI, the pursuit of other possible treatments has intensified over the last years. FMT is one of the potential therapies, which has been used quite often since 2010, primarily due to its good efficacy (in some studies, it has been successful in over $90 \%$ of cases). ${ }^{63,64}$

\section{FMT as a therapy for CDI}

The concept of FMT implies the restoration of the altered microbiota in the intestines (dysbiosis) of the diseased person to a healthy microbiome (symbiosis). This regeneration is performed by transmitting the intestinal flora of a healthy donor to the intestine of an infected person. ${ }^{65}$ As an opportunistic pathogen, $C$ difficile promotes disease when microbiota homeostasis is disrupted. Thus, restoration of balanced intestinal microflora enables suppression of toxic strain of $C$ difficile by anaerobic bacteria of normal intestinal microflora with accompanying cure. ${ }^{66}$

There are three primary indications for FMT in the case of CDI: (1) CDI that reoccurs repeatedly, (2) restrained CDI resistant to standard antibiotic therapy (vancomycin or fidaxomicin) during minimum one week, and (3) serious form of ulcerative colitis (fulminant CDI) not responding to standard antibiotic therapy during 48 hours. ${ }^{67}$ In some cases of CDI, antibiotic therapy is unsuccessful due to increased formation of $C$ difficile toxins, delayed response of immune system or conditions such as ileus or diverticulum, preventing the antibiotic from reaching the colon. FMT can be used as a therapeutic option in these cases, especially if the patient is not a candidate for surgery. ${ }^{68}$ In addition to CDI, this method is used as a therapy for metabolic syndrome, irritable bowel syndrome and inflammatory bowel disease. Research on FMT use in some other diseases, such as Parkinson's disease, multiple sclerosis, autism and obesity, is underway. ${ }^{69,70}$ FMT leads to a more robust immune response, faster healing but also improves the overall quality of life and the FMT procedure is straightforward. ${ }^{71}$

To perform FMT, it is necessary to provide a healthy donor, the equipment of the laboratory required for processing and storage of faeces and medical equipment for the transfer of faeces into the small or large intestine of the patient. Research has shown that donor selection, processing and storage of faeces differ somewhat, but standardized procedures already exist. ${ }^{72}$ FMT providers can be older than 18 years, known to the patient (family members or friends) or unknown, ie those who want to give faeces (volunteers). Family members, especially immediate family, share the most significant amount and content of microorganisms with the recipient. While family members share the risk 
factors for infection diseases as well, the factors of risk are minimised with unknown donors, as they go through the same procedure as blood donors. ${ }^{73}$ In any case, all faecal donors for FMT must undergo rigorous screening to reduce the possibility of transmitting infectious diseases. Current FMT guidelines advise employing a donor questionnaire analogous to the one required for voluntary blood donors, serological testing, or faecal examination for infectious agents. ${ }^{74}$ The donor cannot be a person if he has any of the gastrointestinal diseases (inflammatory bowel disease, irritable bowel syndrome, constipation, chronic diarrhoea, history of major gastrointestinal surgery, gastrointestinal cancer or polyposis) or conditions that may influence the intestinal microbiota setup (metabolic syndrome, systemic autoimmune diseases, atopic diseases). Also, donors should not use antibiotics and immunosuppressive drugs for three months before faecal administration.

After answering the questions from the questionnaire, serological testing for syphilis, hepatitis $A$, hepatitis B, hepatitis $\mathrm{C}$, human immunodeficiency virus (HIV), and human T lymphotropic virus (HTLV) I and II is performed. Donor faeces should be tested for bacteria, viruses, protozoa, and parasites: $C$ difficile, Helicobacter pylori, Listeria spp, Salmonella spp, Shigella spp, Vibrio spp, Yersinia spp, Campylobacter spp, Escherichia coli (E coli) 0157 H7, methicillin-resistant Staphylococcus aureus (MRSA), vancomycin-resistant Enterococcus (VRE) and Gram-negative bacteria producing extended-spectrum $\beta$-lactamases and carbapenemases (ESBL), rotavirus, norovirus, adenovirus. ${ }^{75}$ Some authors recommend a short screening protocol for the donor that is a close relative or intimate partner. In contrast, others suggest that all tests should be performed regardless of the donor's choice. ${ }^{76,77}$ The faecal sample for transplantation can be prepared as fresh, frozen or in the form of gelatine capsules. If it is fresh faeces, it is recommended to be transplanted within six hours of defecation. To preserve anaerobic bacteria, some research also suggests automated preparation of faeces, so that the time of preparation itself is reduced to one hour. Faeces can also be stored at $-80^{\circ} \mathrm{C}$. Before the application, the stool suspension should be heated to $37^{\circ} \mathrm{C}$ in a water bath, and the transfer should be performed within six hours. $^{78,79}$

\section{Processing of donor faeces for FMT}

The faecal sample must be properly taken from an appropriate donor, must not encounter contaminated water, urine or blood, has to be in the amount of at least 50 grams and delivered to the laboratory at shortest convenience. The faeces are homogenised in the laboratory by centrifugation in sterile $0.9 \%$ saline, filtered and resuspended. ${ }^{80}$ How FMT will be performed depends on the clinical presentation but also on the patient's requirements. The methods of faecal transplantation currently used are: 1) endoscopy, via a nasoduodenal probe or by swallowing a gelatine capsule, for the upper part of the gastrointestinal tract, 2) colonoscopy, for the initial part of the colon, 3) sigmoidoscopy, rectal probe or enema, for the final part of the colon. The combined route of administration is preferred for more complex conditions, as for example ileus or anatomical disorders of the gastrointestinal tract. ${ }^{81,82}$

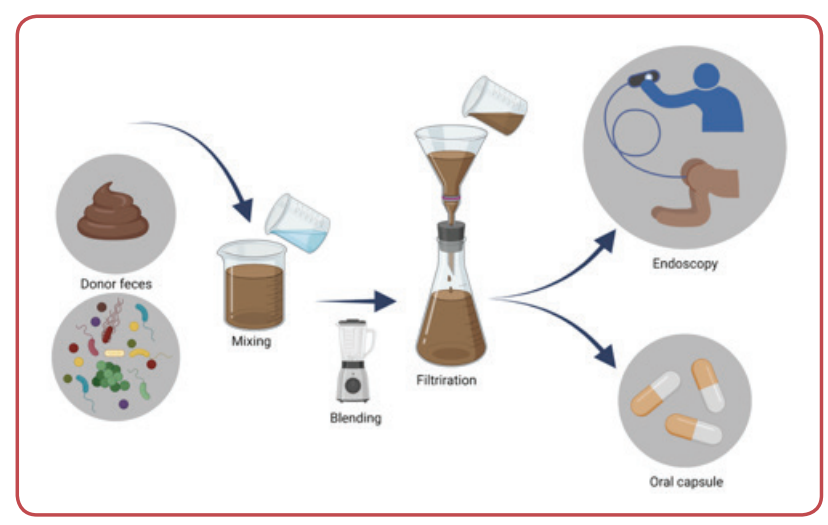

Figure 1: Schematic presentation of faecal microbiota transplantation [Wang et al, 2019]

Although, in general, the FMT procedure is considered safe, possible complications have not been well studied as large cohort studies are still missing. After colonoscopic faecal transplantation, stomach pain, diarrhoea, distension with nausea and vomiting, obstipation and mild fever may occur. Such symptoms are usually temporal and subside after several hours. ${ }^{84}$ Complications of FMT are associated with risks associated with FMT itself, eg exposure to pathogens. Although the overall risk of exposure to pathogens is considered low, pathogens that can be transmitted include $E$ coli and norovirus. ${ }^{85}$ 


\section{The role of the nurse}

Nurses play an essential role in caring for patients with CDI, after they have been subjected to FMT, primarily if those patients are housed in an intensive care unit. Enteric preventive measures and proper isolation of patients with CDI are crucial care procedures. ${ }^{86}$ According to the CDI prevention guidelines, contact isolation must be applied for the duration of the disease. The prescribed protective equipment is used: gloves, surgical mask, protective coat during care, PVC apron during other minor interventions. Protective equipment should also be used by those visiting the patient. Hygienic handwashing with soap after each direct contact with the patient is essential, and disinfectants with alcohol must not be used because they do not have a sporicidal effect. Equipment used to access a patient with suspected CDI or confirmed infection should be disposable or intended for these patients only (stethoscopes, thermometers, sphygmomanometer cuffs). The patient area is thoroughly cleaned and disinfected. Nurses should inform the patient about CDI, and his family and visitors about the importance of implementing prescribed hygiene measures. ${ }^{87}$

Patients prone to developing CDI have often used antimicrobial drugs in the recent past (eg, as a surgical prophylaxis, due to hospitalisation caused by long-term infection, following immunosuppressive therapy or older age). In case the antibiotic therapy is necessary even after FMT, it will again cause a change in the patient's intestinal microbiota. After FMT procedure, nurses should monitor patients who use antibiotics for possible recurrent CDI. ${ }^{88}$ Patients who have undergone FMT are often bedridden and need special care. Since the patient's environment is most likely contaminated with $C$ difficile spores, the patient's room must be thoroughly cleaned with a chlorine-containing agent immediately before performing FMT. If technically feasible, the patient should be placed in a new or thoroughly cleaned bed with clean bedding. Reducing the number of $C$ difficile spores improves the success of FMT administration in patients with CDI. The patient should be asked not to defecate for at least 4 hours, if he can endure, after performing FMT, and if he can go for longer, he must be placed in a semi-sitting position for 24 hours and given diarrhoea medication. ${ }^{89}$

\section{Conclusion}

By modulating an individual microbiome, many diseases can be prevented and treated. One of the latest approaches to treating recurrent CDI cases is the FMT method. It is a simple method during which the faeces of a healthy donor is being transplanted to a patient. Several faecal transplant procedures are used, and the most important thing is to perform safe preparation. Nurses apply specific prevention measures during the care of patients who have undergone FMT. In order to improve the effectiveness of FMT in patients with CDI, it is necessary to ask them not to defecate by placing them in a semi-sitting position.

\section{Acknowledgements}

None.

\section{Conflict of interest}

None.

\section{References}

1. Rossen NG, MacDonald JK, de Vries EM, D'Haens GR, de Vos WM, Zoetendal EG, et al. Fecal microbiota transplantation as novel therapy in gastroenterology: A systematic review. World J Gastroenterol 2015;21(17):5359-71.

2. Kelly BJ, Tebas P. Clinical practice and infrastructure review of fecal microbiota transplantation for Clostridium difficile infection. Chest 2018 Jan;153(1):266-77.

3. Bakker GJ, Nieuwdorp M. Fecal microbiota transplantation: therapeutic potential for a multitude of diseases beyond Clostridium difficile. Microbiol Spectr 2017 Aug;5(4). doi: 10.1128/microbiolspec.BAD-0008-2017.

4. Du H, Kuang TT, Qiu S, Xu T, Gang Huan CL, Fan G, et al. Fecal medicines used in traditional medical system of China: a systematic review of their names, original species, traditional uses, and modern investigations. Chin Med 2019 Sep 13;14:31. doi: 10.1186/s13020-019-0253-x.

5. Zhang F, Luo W, Shi Y, Fan Z, Ji G. Should we standardize the 1,700-year-old fecal microbiota transplantation? Am J Gastroenterol 2012;107(11):1755-6.

6. Eiseman B, Silen W, Bascom GS, Kauvar AJ. Fecal enema as an adjunct in the treatment of pseudomembranous enterocolitis. Surgery 1958;44(5):854-9.

7. Rossen NG, Fuentes S, van der Spek MJ, Tijssen JG, Hartman JHA, Duflou A, et al. Findings from a randomized controlled trial of fecal transplantation for patients with ulcerative colitis. Gastroenterology 2015 Jul;149(1):110-8. 
8. Paramsothy S, Paramsothy R, Rubin DT, Kamm MA, Kaakoush NO, Mitchell HM, et al. Faecal microbiota transplantation for inflammatory bowel disease: a systematic review and meta-analysis. J Crohns Colitis 2017 Oct;11(10):1180-99.

9. Marotz CA, Zarrinpar A. Treating obesity and metabolic syndrome with fecal microbiota transplantation. Yale J Biol Med 2016;89(3):383-8.

10. Gerard P. Gut microbiota and obesity. Cell Mol Life Sci 2016;73(1):147-62.

11. Qin J, Li R, Raes J, Arumugam M, Burgdorf KS, Manichanh $\mathrm{C}$, et al. A human gut microbial gene catalogue established by metagenomic sequencing. Nature 2010;464(7285):5965.

12. Antal I, Jelić MS, Sila S, Kolaček A, Tambić Andrašević A. [Human microbiota and microbiome]. Acta Med Croatica 2019;(73):3-11. Croatian.

13. Goodrich JK, Davenport ER, Clark AG, Ley RE. The relationship between the human genome and microbiome comes into view. Annu Rev Genet 2017;51:413-33.

14. Shreiner AB, Kao JY, Young VB. The gut microbiome in health and in disease. Curr Opin Gastroenterol 2015;31(1):69-75.

15. Malikowski T, Khanna S, Pardi DS. Fecal microbiota transplantation for gastrointestinal disorders. Curr Opin Gastroenterol 2017 Jan;33(1):8-13.

16. Kowalska-Duplaga K, Gosiewski T, Kapusta P, Sroka-Oleksiak A, Wędrychowicz A, Pieczarkowski S, et al. Differences in the intestinal microbiome of healthy children and patients with newly diagnosed Crohn's disease. Sci Rep 2019 Dec 11;9(1):18880. doi: 10.1038/s41598-019-55290-9.

17. Collado MC, Rautava S, Aakko J, Isolauri E, Salminen S. Human gut colonisation may be initiated in utero by distinct microbial communities in the placenta and amniotic fluid. Sci Rep 2016 Mar 22;6:23129. doi: 10.1038/srep23129.

18. Xiao L, Ding G, Ding Y, Deng C, Ze X, Chen L, et al. Effect of probiotics on digestibility and immunity in infants: A study protocol for a randomized controlled trial. Medicine (Baltimore) 2017 Apr;96(14):e5953. doi: 10.1097/ MD.0000000000005953.

19. Lee N, Kim WU. Microbiota in T-cell homeostasis and inflammatory diseases. Exp Mol Med 2017 May 26;49(5):e340. doi: 10.1038/emm.2017.36.

20. Chen X, Devaraj S. Gut microbiome in obesity, metabolic syndrome, and diabetes. Curr Diab Rep 2018 Oct 18;18(12):129. doi: 10.1007/s11892-018-1104-3.

21. Guinane CM, Cotter PD. Role of the gut microbiota in health and chronic gastrointestinal disease: understanding a hidden metabolic organ. Therap Adv Gastroenterol 2013;6(4):295-308.

22. Hollister EB, Gao C, Versalovic J. Compositional and functional features of the gastrointestinal microbiome and their effects on human health. Gastroenterology 2014;146(6):1449-58.

23. Shreiner $A B$, Kao JY, Young VB. The gut microbiome in health and in disease. Curr Opin Gastroenterol 2015;31(1):69-75.

24. Moles L, Otaegui D. The impact of diet on microbiota evolution and human health. is diet an adequate tool for microbiota modulation? Nutrients 2020 Jun 2;12(6):1654. doi: $10.3390 /$ nu12061654.

25. Manson JM, Rauch M, Gilmore MS. The commensal microbiology of the gastrointestinal tract. Adv Exp Med Biol 2008;635:15-28.

26. Ventura M, Turroni F, Canchaya C, Vaughan EE, O’Toole $\mathrm{PW}$, van Sinderen D. Microbial diversity in the human intestine and novel insights from metagenomics. Front Biosci 2009;14:3214-21.

27. Jandhyala SM, Talukdar R, Subramanyam C, Vuyyuru H, Sasikala M, Nageshwar Reddy D. Role of the normal gut microbiota. World J Gastroenterol 2015;21(29):8787-803.

28. Larsbrink J, Rogers TE, Hemsworth GR, McKee LS, Tauzin AS, Spadiut 0, et al. A discrete genetic locus confers xyloglucan metabolism in select human gut Bacteroidetes. Nature 2014;506(7489):498-502.

29. Wang BY, Mingfei L, Longxian L, Zongxin L. The human microbiota in health and disease. Engineering 2017;3:71-82.

30. Scott AJ, Alexander JL, Merrifield CA, Cunningham D, Jobin $\mathrm{C}$, Brown R, et al. International Cancer Microbiome Consortium consensus statement on the role of the human microbiome in carcinogenesis. Gut 2019;68(9):1624-32.

31. Khan I, Ullah N, Zha L, Bai Y, Khan A, Zhao T, et al. Alteration of gut microbiota in inflammatory bowel disease (IBD): cause or consequence? IBD treatment targeting the gut microbiome. Pathogens 2019 Aug 13;8(3):126. doi: 10.3390/pathogens8030126.

32. Rautava S, Luoto R, Salminen S, Isolauri E. Microbial contact during pregnancy, intestinal colonization and human disease. Nat Rev Gastroenterol Hepatol 2012;9:565-76.

33. Rogier EW, Frantz AL, Bruno ME, Wedlund L, Cohen DA, Stromberg AJ, et al. Lessons from mother: long-term impact of antibodies in breast milk on the gut microbiota and intestinal immune system of breastfed offspring. Gut Microbes 2014;5(5):663-8.

34. Clemente JC, Ursell LK, Parfrey LW, Knight R. The impact of the gut microbiota on human health: an integrative view. Cell 2012;148(6):1258-70.

35. Sorbara MT, Pamer EG. Interbacterial mechanisms of colonization resistance and the strategies pathogens use to overcome them [published correction appears in $\mathrm{Mu}$ cosal Immunol 2019 May;12(3):840]. Mucosal Immunol 2019;12(1):1-9.

36. Abt MC, McKenney PT, Pamer EG. Clostridium difficile colitis: pathogenesis and host defence. Nat Rev Microbiol 2016;14:609-20.

37. Ling Z, Liu X, Jia X, Cheng Y, Luo Y, Yuan L, et al. Impacts of infection with different toxigenic Clostridium difficile strains on faecal microbiota in children. Sci Rep 2014;4:7485. doi: 10.1038/srep07485.

38. Collins J, Auchtung JM. Control of Clostridium difficile infection by defined microbial communities. Microbiol Spectr 2017 Sep;5(5):10.1128/microbiolspec.BAD-00092016. doi: 10.1128/microbiolspec.BAD-0009-2016.

39. Cohen J. Vaginal microbiome affects HIV risk. Science 2016;353(6297):331.

40. Xu M, Wang B, Fu Y, Chen Y, Yang F, Lu H, et al. Changes of fecal Bifidobacterium species in adult patients with hepatitis $B$ virus-induced chronic liver disease. Microb Ecol 2012;63(2):304-13.

41. Hu Z, Zhang Y, Li Z, Yu Y, Kang W, Han Y, et al. Effect of Helicobacter pylori infection on chronic periodontitis by the change of microecology and inflammation. Oncotarget 2016;7(41):66700-12.

42. Rao K, Micic D, Natarajan M, Winters S, Kiel MJ, Walk ST, et al. Clostridium difficile ribotype 027: relationship to age, detectability of toxins A or B in stool with rapid testing, severe infection, and mortality. Clin Infect Dis 2015 Jul 15;61(2):233-41.

43. Ghose C. Clostridium difficile infection in the twenty-first century. Emerg Microbes Infect 2013;2:e62. doi: 10.1038/ emi.2013.62.

44. Slimings C, Riley TV. Antibiotics and hospital-acquired Clostridium difficile infection: update of systematic review and meta-analysis. J Antimicrob Chemother 2014;69:881-91.

45. Lessa FC, Winston LG, McDonald LC. Emerging infections program C. difficile surveillance team, burden of Clostridium difficile infection in the united states. $\mathrm{N}$ Engl J Med 2015;372:2369-70.

46. Lawson PA, Citron DM, Tyrrell KL, Finegold SM. Reclas- 
sification of Clostridium difficile as Clostridioides difficile (Hall and O'Toole 1935) Prévot 1938. Anaerobe 2016 Aug;40:95-9.

47. Smits WK, Lyras D, Lacy DB, Wilcox MH, Kuijper EJ. Clostridium difficile infection. Nat Rev Dis Primers 2016;2:16020.

48. Zacharioudakis IM, Zervou FN, Pliakos EE, Ziakas PD, Mylonakis E. Colonization with toxinogenic C. difficile upon hospital admission, and risk of infection: a systematic review and meta-analysis. Am J Gastroenterol 2015 Mar;110(3):381-90.

49. Rupnik M. Is Clostridium difficile-associated infection a potentially zoonotic and foodborne disease? Clin Microbiol Infect 2007;13:457-9.

50. Warriner K, Xu C, Habash M, Sultan S, Weese SJ. Dissemination of Clostridium difficile in food and the environment: Significant sources of C. difficile community-acquired infection? J Appl Microbiol 2017 Mar;122(3):542-53.

51. Loo VG. Environmental interventions to control Clostridium difficile. Infect Dis Clin North Am 2015;29(1):83-91.

52. Vardakas KZ, Trigkidis KK, Boukouvala E, Falagas ME. Clostridium difficile infection following systemic antibiotic administration in randomised controlled trials: a systematic review and meta-analysis. Int J Antimicrob Agents 2016;48:1-10.

53. Jangi S, Lamont JT. Asymptomatic colonization by Clostridium difficile in infants: implications for disease in later life. J Pediatr Gastroenterol Nutr 2010;51(1):2-7.

54. Koenig JE, Spor A, Scalfone N, Fricker AD, Stombaugh J, Knight R, et al. Succession of microbial consortia in the developing infant gut microbiome. Proc Natl Acad Sci U S A 2011 Mar 15;108 Suppl 1(Suppl 1):4578-85.

55. Bäckhed F, Roswall J, Peng Y, Feng Q, Jia H, Kovatcheva-Datchary $\mathrm{P}$, et al. Dynamics and stabilization of the human gut microbiome during the first year of life. Cell Host Microbe 2015 Jun 10;17(6):852. doi: 10.1016/j. chom.2015.05.012.

56. Scardina T, Labuszewski L, Pacheco SM, Adams W, Schreckenberger P, Johnson S. Clostridium difficile infection (CDI) severity and outcome among patients infected with the NAP1/BI/027 strain in a non-epidemic setting. Infect Control Hosp Epidemiol 2015;36(3):280-6.

57. Furuya-Kanamori L, Marquess J, Yakob L, Riley TV, Paterson DL, Foster NF, et al. Asymptomatic Clostridium difficile colonization: epidemiology and clinical implications. BMC Infect Dis 2015 Nov 14;15:516. doi: 10.1186/s12879015-1258-4.

58. Cohen SH, Gerding DN, Johnson S, Kelly CP, Loo VG, McDonald LC, et al. Society for Healthcare Epidemiology of America; Infectious Diseases Society of America. Clinical practice guidelines for Clostridium difficile infection in adults: 2010 update by the society for healthcare epidemiology of America (SHEA) and the infectious diseases society of America (IDSA). Infect Control Hosp Epidemiol 2010 May;31(5):431-55.

59. Caroff DA, Edelstein PH, Hamilton K, Pegues DA. The Bristol Stool Scale and its relationship to Clostridium difficile infection. J Clin Microbiol 2014;52(9):3437-9.

60. Olsen MA, Yan Y, Reske KA, Zilberberg MD, Dubberke ER. Recurrent Clostridium difficile infection is associated with increased mortality. Clin Microbiol Infect 2015;21(2):164-70.

61. Zilberberg MD, Reske K, Olsen M, Yan Y, Dubberke ER. Risk factors for recurrent Clostridium difficile infection (CDI) hospitalization among hospitalized patients with an initial CDI episode: a retrospective cohort study. BMC Infect Dis 2014;14(1):306. doi: 10.1186/1471-2334-14-306.

62. Dieterle MG, Rao K, Young VB. Novel therapies and preventative strategies for primary and recurrent Clostridium difficile infections. Ann N Y Acad Sci 2019;1435(1):110-38.
63. van Nood E, Vrieze A, Nieuwdorp M, Fuentes S, Zoetendal EG, de Vos WM, et al. Duodenal infusion of donor feces for recurrent Clostridium difficile. N Engl J Med 2013 Jan 31;368(5):407-15.

64. Bobo L. Fecal microbial transplantation: highly effective treatment for severe Clostridium difficile infection. ID Week 2013;281-4.

65. Filip M, Tzaneva V, Dumitrascu DL. Fecal transplantation: digestive and extradigestive clinical applications. Clujul Med 2018;91(3):259-65.

66. Theriot CM, Young VB. Interactions between the gastrointestinal microbiome and Clostridium difficile. Annu Rev Microbiol 2015;69:445-61.

67. Bakken JS, Borody T, Brandt LJ, Brill JV, Demarco DC, Franzos MA, et al. Fecal Microbiota Transplantation Workgroup. Treating Clostridium difficile infection with fecal microbiota transplantation. Clin Gastroenterol Hepatol 2011 Dec;9(12):1044-9..

68. Weingarden AR, Hamilton MJ, Sadowsky MJ, Khoruts A. Resolution of severe Clostridium difficile infection following sequential fecal microbiota transplantation. J Clin Gastroenterol 2013;47(8):735-7.

69. Kelly CR, Kahn S, Kashyap P, Laine L, Rubin D, Atreja A, et al. Update on fecal microbiota transplantation 2015: indications, methodologies, mechanisms, and outlook. Gastroenterology 2015 Jul;149(1):223-37.

70. Choi HH, Cho YS. Fecal microbiota transplantation: current applications, effectiveness, and future perspectives. Clin Endosc 2016;49(3):257-65.

71. Distrutti E, Monaldi L, Ricci P, Fiorucci S. Gut microbiota role in irritable bowel syndrome: New therapeutic strategies. World J Gastroenterol 2016;22(7):2219-41.

72. Kelly BJ, Tebas P. Clinical practice and infrastructure review of fecal microbiota transplantation for Clostridium difficile infection. Chest 2018 Jan;153(1):266-77.

73. Lagier JC. Faecal microbiota transplantation: from practice to legislation before considering industrialization. Clin Microbiol Infect 2014;20:1112-8.

74. Dorsey KA, Moritz ED, Steele WR, Eder AF, Stramer SL. A comparison of human immunodeficiency virus, hepatitis $\mathrm{C}$ virus, hepatitis $\mathrm{B}$ virus, and human T-lymphotropic virus marker rates for directed versus volunteer blood donations to the American Red Cross during 2005 to 2010. Transfusion 2013;53(6):1250-6.

75. Reigadas E, Olmedo M, Valerio M, Vázquez-Cuesta S, Alcalá L, Marín M, et al. Fecal microbiota transplantation for recurrent Clostridium difficile infection: Experience, protocol, and results. Rev Esp Quimioter 2018 Oct;31(5):411-8.

76. Hamilton MJ, Weingarden AR, Sadowsky MJ, Khoruts A. Standardized frozen preparation for transplantation of fecal microbiota for recurrent Clostridium difficile infection. Am J Gastroenterol 2012;107(5):761-7.

77. Kazerouni A, Burgess J, Burns LJ, Wein LM. Optimal screening and donor management in a public stool bank. Microbiome 2015;3(1):75. doi: 10.1186/s40168-0150140-3.

78. Cui B, Li P, Xu L, Peng Z, Xiang J, He Z, et al. Step-up fecal microbiota transplantation (FMT) strategy. Gut Microbes 2016;7:323-8

79. Cammarota G, Ianiro G, Tilg H, Rajilić-Stojanović M, Kump $\mathrm{P}$, Satokari R, et al. European consensus conference on faecal microbiota transplantation in clinical practice. Gut 2017;66(4):569-80.

80. Zhang F, Cui B, He X, Nie Y, Wu K, Fan D; FMT-standardization Study Group. Microbiota transplantation: concept, methodology and strategy for its modernization. Protein Cell 2018 May;9(5):462-73.

81. Choi HH, Cho YS. Fecal microbiota transplantation: current applications, effectiveness, and future perspectives. Clin Endosc 2016;49(3):257-65. 
82. Reigadas E, Olmedo M, Valerio M, Vázquez-Cuesta S, Alcalá L, Marín M, et al. Fecal microbiota transplantation for recurrent Clostridium difficile infection: Experience, protocol, and results. Rev Esp Quimioter 2018 Oct;31(5):411-8.

83. Wang JW, Kuo CH, Kuo FC, Wang YK, Hsu WH, Yu FJ, et al. Fecal microbiota transplantation: Review and update. J Formos Med Assoc 2019 Mar;118 Suppl 1:S23-S31. doi: 10.1016/j.jfma.2018.08.011.

84. Cammarota G, Ianiro G, Gasbarrini A. Fecal microbiota transplantation for the treatment of Clostridium difficile infection: a systematic review. J Clin Gastroenterol 2014;48(8):693-702.

85. Liubakka A, Vaughn BP. Clostridium difficile infection and fecal microbiota transplant. AACN Adv Crit Care 2016;27(3):324-37.

86. Guillemin I, Marrel A, Beriot-Mathiot A, Doucet C, Kazoglou 0 , Luxemburger C, et al. How do Clostridium difficile infections affect nurses' everyday hospital work: A qualitative study. Int J Nurs Pract 2015 May;21 Suppl 2:38-45.

87. McDonald LC, Gerding DN, Johnson S, Bakken JS, Carroll KC, Coffin SE, et al. Clinical Practice Guidelines for Clostridium difficile Infection in Adults and Children: 2017 Update by the Infectious Diseases Society of America (IDSA) and Society for Healthcare Epidemiology of America (SHEA). Clin Infect Dis 2018 Mar 19;66(7):987-94.

88. Walton J, Burns D, Gaehle KE. Process and outcome of fecal microbiota transplants in patients with recurrent Clostridium difficile infection: a prospective study. Gastroenterol Nurs 2017 Sep/Oct;40(5):411-9.

89. Rohlke F, Surawicz CM, Stollman N. Fecal flora reconstitution for recurrent Clostridium difficile infection: results and methodology. J Clin Gastroenterol 2010 Sep;44(8):567-70. 